\title{
POLR1B and neural crest cell anomalies in Treacher Collins syndrome type 4
}

\author{
Elodie Sanchez, $\mathrm{MLT}^{1,2}$, Béryl Laplace-Builhé, $\mathrm{PhD}^{2}$, Frédéric Tran Mau-Them, MD 1,2,3,4, \\ Eric Richard, PhD ${ }^{5}$, Alice Goldenberg, $\mathrm{MD}^{6}$, Tomi L. Toler, $\mathrm{MS}^{7}$, Thomas Guignard, $\mathrm{PhD}^{8}$, \\ Vincent Gatinois, PharmD, PhD ${ }^{8}$, Marie Vincent, $\mathrm{MD}^{9}$, Catherine Blanchet, MD ${ }^{10}$, Anne Boland, $\mathrm{PhD}^{11}$, \\ Marie Thérèse Bihoreau, $\mathrm{MLT}^{11}$, Jean-Francois Deleuze, $\mathrm{PhD}^{11}$, Robert Olaso, $\mathrm{PhD}^{11}$, \\ Walton Nephi, MD", Hermann-Josef Lüdecke, MD ${ }^{12}$, Joke B. G. M. Verheij, MD ${ }^{13}$, \\ Florence Moreau-Lenoir, MD, $\mathrm{PhD}^{14}$, Françoise Denoyelle, $\mathrm{MD}, \mathrm{PhD}^{15}$, Jean-Baptiste Rivière, $\mathrm{PhD}^{16}$, \\ Jean-Louis Laplanche, PharmD, $\mathrm{PhD}^{17}$, Marcia Willing, $\mathrm{MD}^{7}$, Guillaume Captier, $\mathrm{MD}, \mathrm{PhD}^{18}$, \\ Florence Apparailly, PhD ${ }^{2}{ }^{2}$, Dagmar Wieczorek, MD, PhD ${ }^{12}$, Corinne Collet, PharmD, PhD ${ }^{17}$, \\ Farida Djouad, $\mathrm{PhD}^{2}$ and David Geneviève, $\mathrm{MD}, \mathrm{PhD}^{1,2}$
}

Purpose: Treacher Collins syndrome (TCS) is a rare autosomal dominant mandibulofacial dysostosis, with a prevalence of $0.2-1$ / 10,000. Features include bilateral and symmetrical malar and mandibular hypoplasia and facial abnormalities due to abnormal neural crest cell (NCC) migration and differentiation. To date, three genes have been identified: TCOF1, POLR1C, and POLR1D. Despite a large number of patients with a molecular diagnosis, some remain without a known genetic anomaly.

Methods: We performed exome sequencing for four individuals with TCS but who were negative for pathogenic variants in the known causative genes. The effect of the pathogenic variants was investigated in zebrafish.

Results: We identified three novel pathogenic variants in POLR1B. Knockdown of polr $1 b$ in zebrafish induced an abnormal craniofacial phenotype mimicking TCS that was associated with altered ribosomal gene expression, massive p53-associated cellular apoptosis in the neuroepithelium, and reduced number of NCC derivatives.

Conclusion: Pathogenic variants in the RNA polymerase I subunit POLR1B might induce massive p53-dependent apoptosis in a restricted neuroepithelium area, altering NCC migration and causing cranioskeletal malformations. We identify $P O L R 1 B$ as a new causative gene responsible for a novel TCS syndrome (TCS4) and establish a novel experimental model in zebrafish to study POLR1B-related TCS.

Genetics in Medicine (2020) 22:547-556; https://doi.org/10.1038/s41436019-0669-9

Keywords: Treacher Collins-Franceschetti; POLR1B; apoptosis; neural crest cells

\section{INTRODUCTION}

Treacher Collins-Franceschetti syndrome (TCS; TCS1 [OMIM 154500], TCS2 [OMIM 613717], TCS3 [OMIM 248390]) is a rare disorder of craniofacial development with an estimated incidence of 1/50,000 live births. ${ }^{1}$ TCS belongs to a large group of disorders called mandibulofacial dysostosis (MFD). Features of TCS include bilateral malar and mandibular hypoplasia with associated facial features (microtia, coloboma, and downslanting palpebral fissures) and other features such as conductive deafness, cleft palate, and heart malformations. Facial features in TCS are due to abnormal neural crest cell (NCC) migration secondary to nucleolar stress, decreased ribosome biogenesis, and apoptosis. ${ }^{2,3}$ Therefore, TCS belongs to the neurocristopathies and ribosomopathies, a large group of disorders.

\footnotetext{
${ }^{1}$ Service de Génétique Clinique, centre de référence anomalies du développement et syndromes malformatifs, Département de Génétique Médicale, Maladies Rares et Médecine Personnalisée, Hôpital Arnaud de Villeneuve, Faculté de Médecine, Montpellier, France; ${ }^{2}$ IRMB, Univ Montpellier, INSERM, CHU Montpellier, Montpellier, France; ${ }^{3}$ Unité Fonctionnelle d'Innovation diagnostique des maladies rares, Pôle de Biologie, FHU-TRANSLAD, CHU Dijon Bourgogne, Dijon, France; ${ }^{4}$ Inserm-Université de Bourgogne UMR1231 GAD, FHU-TRANSLAD, Dijon, France; ${ }^{5}$ IRCM, INSERM, U1194 Univ Montpellier, Montpellier, France; ${ }^{6}$ Normandie Univ, UNIROUEN, Inserm U1245 and Rouen University Hospital, Department of Genetics, F 76000, Normandy Center for Genomic and Personalized Medicine, Reference Center for Developmental Disorders, Rouen, France; ${ }^{7}$ Department of Pediatrics, Division of Genetics \& Genomic Medicine, Washington University School of Medicine, St. Louis, MO, USA; ${ }^{8}$ Unité de Génétique Chromosomique, Plateforme ChromoStem, Hôpital Arnaud de Villeneuve, CHU Montpellier, Montpellier, France; ${ }^{9}$ Service de génétique médicale, CHU de Nantes, Nantes, France; ${ }^{10}$ Service ORL, Montpellier, France; ${ }^{11}$ Centre National de Recherche en Génomique Humaine (CNRGH), Institut de Biologie François Jacob, CEA, Université Paris-Saclay, Evry, France; ${ }^{12}$ Institute of Human Genetics, Medical Faculty, Heinrich Heine University, Düsseldorf, Germany; ${ }^{13}$ Department of Medical Genetics, University Medical Centre Groningen, University of Groningen, Groningen, The Netherlands; ${ }^{14}$ ORL CH d'Evreux, Évreux, France; ${ }^{15}$ Service d'ORL pédiatrique, Hôpital Universitaire Necker-Enfants Malades, APHP et François Jacob, CEA, Université Paris-Saclay, Evry, France; ${ }^{16}$ Laboratoire de Génétique Moléculaire, Plateau technique de Biologie - CHU Dijon, Dijon, France; ${ }^{17}$ UF de Génétique Moléculaire, Service de Biochimie et Biologie Moléculaire, Hôpital Lariboisière, Paris, France; ${ }^{18}$ Chirurgie plastique infantile Montpellier, Montpellier, France.

Correspondence: Florence Apparailly (florence.apparailly@inserm.fr)

These authors contributed equally: Elodie Sanchez, Béryl Laplace-Builhé, Farida Djouad, David Geneviève
} 
To date, three genes are known to be involved in TCS: treacle ribosome biogenesis factor 1 (TCOF1; OMIM 606847), ${ }^{4}$ RNA polymerase I (pol I) subunit C (POLR1C, OMIM 610060), and RNA pol I subunit D (POLR1D, OMIM 613715). ${ }^{5}$ Pathogenic variants in TCOF1 are responsible for about $80-85 \%$ of TCS cases with typical facial features, whereas POLR1C and POLR1D are involved in less than $10 \%{ }^{6}$

However, despite a large number of TCS individuals with a molecular diagnosis, some remain without a known genetic anomaly. We took advantage of a large series of TCS patients without pathogenic variants in the three known genes responsible for TCS or other known MFD genes. ${ }^{6}$ We performed exome sequencing to identify novel pathogenic variants and/or genes and in vivo functional studies in zebrafish to try to elucidate the underlying pathophysiological mechanisms.

\section{MATERIALS AND METHODS}

\section{Patients}

From a large cohort of 146 patients with TCS previously reported, ${ }^{6}$ we identified 6 without a pathogenic variant or causative copy-number variation in the genes involved in MFD. To try to identify the precise molecular defect in these 6 TCS patients without a molecular diagnosis $(6 / 146,4 \%)$, we performed trio exome sequencing in 4 of the 6 patients and their parents. After identifying a candidate gene in 2 of the 4 patients $(2 / 146,1.3 \%)$ and in 1 patient's mother, we then gathered 3 additional patients via international collaboration (using GeneMatcher ${ }^{7}$ for 1 patient). Clinical data are summarized in Table $\mathbf{1}$.

\section{Exome sequencing}

Nucleotide variations in the POLR1B gene (NM_019014.5) were all identified by using Agilent/Illumina exome sequencing. Sanger sequencing was used for sequence validation and segregation analyses of genetic variations identified in POLR1B (details are in the Supplemental data).

\section{D modeling}

The 3D model of $\mathrm{A} 135 / \mathrm{Rpb} 2$ was designed using homology modeling methods. The human variant sequences of POLR1B (p.[Ser682Arg], p.[Arg1003Cys], and p.[Arg1003Ser]) were searched in the online server ATOME2. This server uses Protein Data Bank (PDB) to find the best structure that fits the variant sequence. It introduces constraints, constructs a model, and refines the model. Our model is based on subchain B (A135/Rpb2) of the crystal structure of the Saccharomyces cerevisiae 14-subunit RNA polymerase (PDB 4C3I). ${ }^{8}$ Human POLR1B and the yeast homolog have strong sequence identity, $44 \%$, and probably share the same function in their complex. We did not use refinements with molecular dynamics for all other subchains because we wanted to assess only the possible perturbations of interactions due to those pathogenic variants.

\section{Ethics statement}

Exome sequencing was performed as a clinical test (diagnosis not research) and thus institutional review board (IRB) approval was not required. All parents or legal guardians gave written informed consent for genetic diagnosis procedures according to the ethics committee of our institution. Informed consent for publication of images was obtained from five of six patients. The experiments performed on zebrafish were conducted as described previously ${ }^{9}$ at the University of Montpellier according to European Union guidelines for handling laboratory animals and the Direction Sanitaire et Vétérinaire de l'Hérault and Comité d'Ethique under reference CEEA-LR-13007.

\section{Zebrafish line and morpholino injections}

Experiments involved the $\mathrm{AB}$ zebrafish strain (ZIRC), transgenic $\operatorname{Tg}(\text { foxd3:GFP })^{10}$ and $\operatorname{Tg}(\text { sox 10:GFP })^{11}$ to visualize NCCs, and transgenic $T g(\text { col2a:mcherry })^{12}$ to visualize cartilage. Embryos were obtained from pairs of adult fish by natural spawning and were raised at $28.5^{\circ} \mathrm{C}$ in tank water. Embryos and larvae were staged as described. ${ }^{13}$ For morpholino injections only embryos were used. To obtain enough eggs, 20 pairs of adult zebrafish were used for the breeding. When possible, we bred two different transgenic lines-(Tg[Foxd3: GFP] and Tg[col2a:mcherry]-to maximize information gathered from the experiments. For imaging and quantitative reverse transcription polymerase chain reaction (RT-qPCR) experiments, 20 embryos were injected per condition: 5 embryos were used for imaging and phenotypic screening, and 15 pooled to ensure enough RNA material for RT-qPCR analyses. Experiments were repeated six times to obtain biological replicates. No statistical methods were used to calculate sample size and no randomization method was used to determine how samples were allocated to experimental groups and processed. No blinding was done.

For polr1b (NM_200518.2) loss-of-function experiments, we used morpholino antisense oligonucleotides (Gene Tools, Philomath, USA) that bind to the region surrounding the translation start site of polr $1 b$ and block translation by steric hindrance (MO zPOLR1B-ATG, here called MO polr1b): 5' TTGCACCACTTGGAGAGATCCATGC 3’. Briefly, 2 nl MO polr1b (1 mM) or control morpholino (5' AATCACAAG CAGTGCAAGCATGATG 3', $1 \mathrm{mM}$ ) was injected in one cell-stage embryos by using a FemtoJet (Eppendorf).

\section{Imaging and larvae manipulation}

Larvae were anesthetized with $0.016 \%$ Tricaine (ethyl 3aminobenzoate, Sigma Aldrich), mounted in 1\% low melting temperature agarose in a FluoroDish and imaged by using confocal inverted microscopy (TCS SP5) with an MVX10 Olympus microscope equipped with an MVPLAPO $1 \times$ objective and XC50 camera.

\section{Terminal deoxynucleotidyl transferase dUTP nick end labeling (TUNEL) experiment}

At 20 hours postfertilization (hpf), MO control- and $\mathrm{MO}$ polr1b-injected $\mathrm{Tg}$ (sox10:GFP) embryos were fixed with PFA $4 \%$ at $4{ }^{\circ} \mathrm{C}$ overnight, rinsed extensively with PBS-Tween 


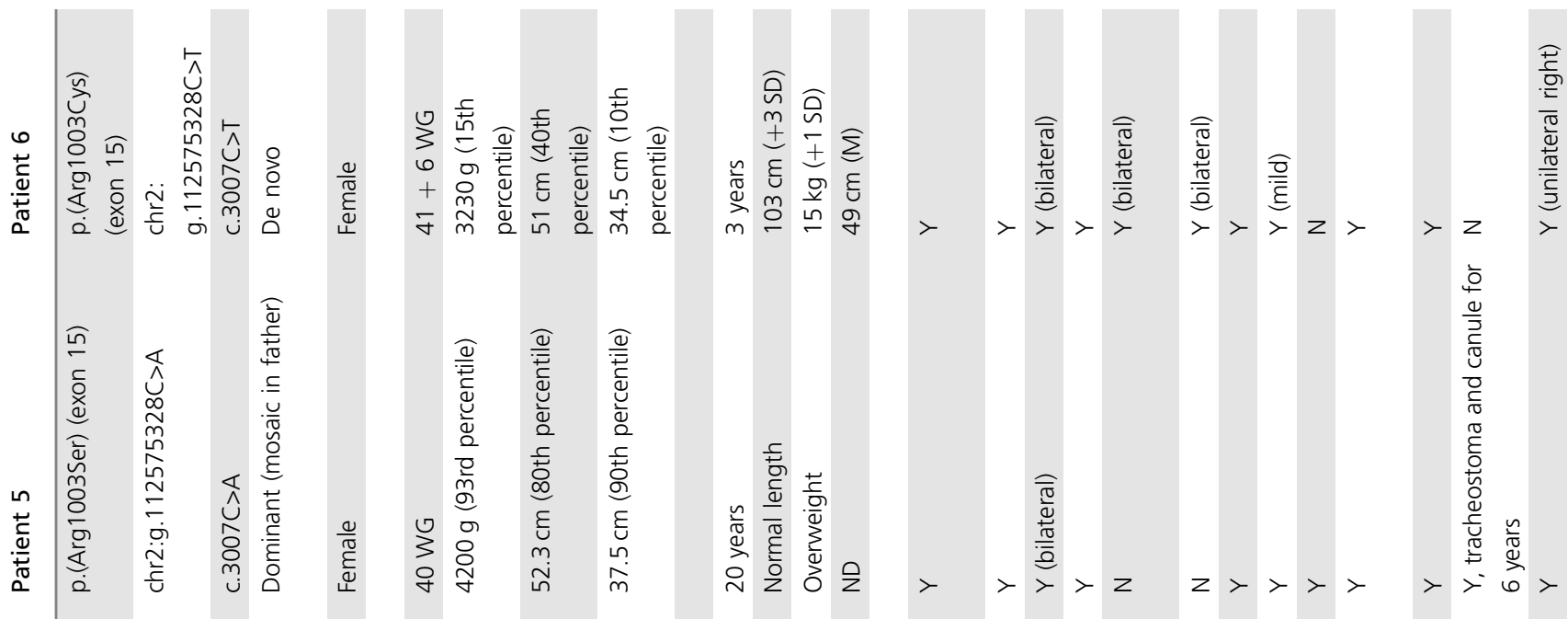

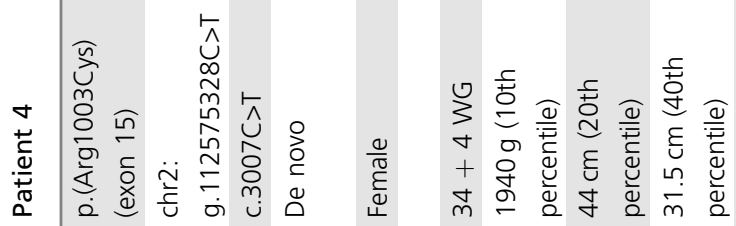
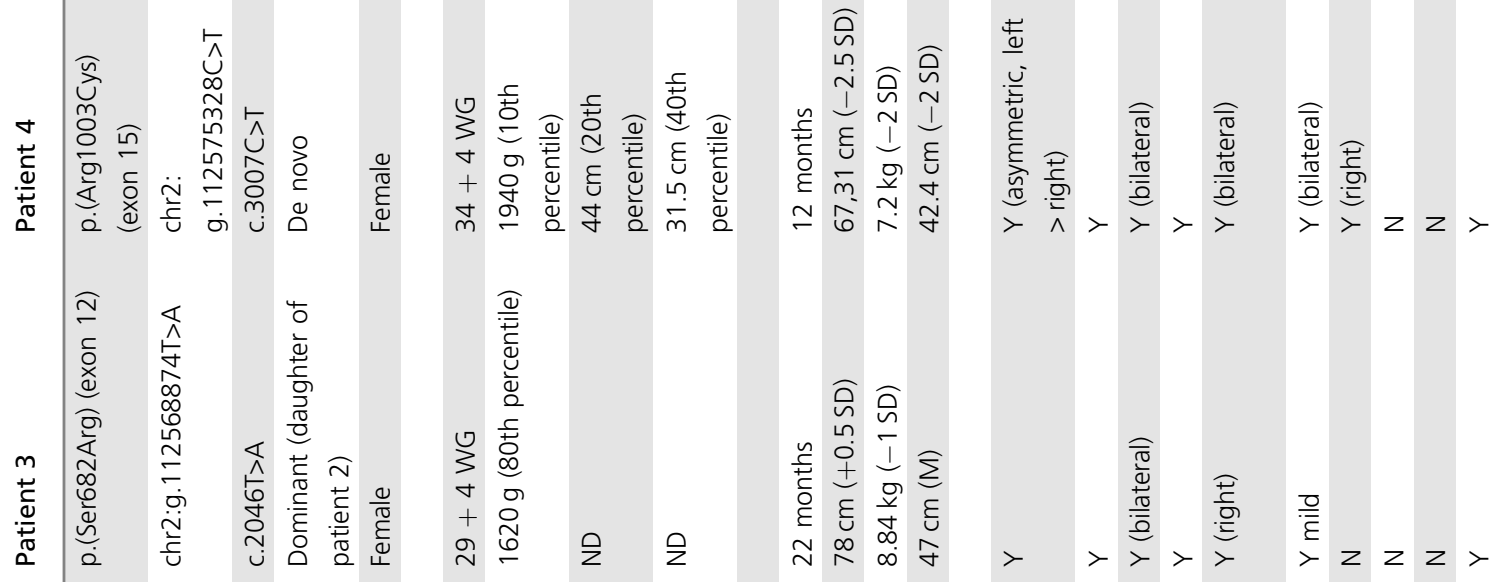

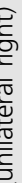

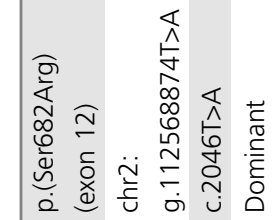

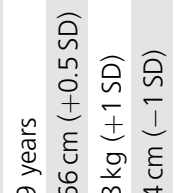

$\frac{5}{3}$

范

范 $\quad \stackrel{\hat{n}}{\infty}$

$\underset{\substack{\infty \\ \sim}}{\sim}$

ลิ $\widehat{\hat{~}} \widehat{ล}$

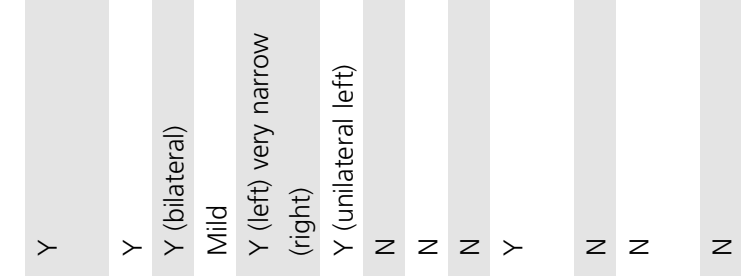




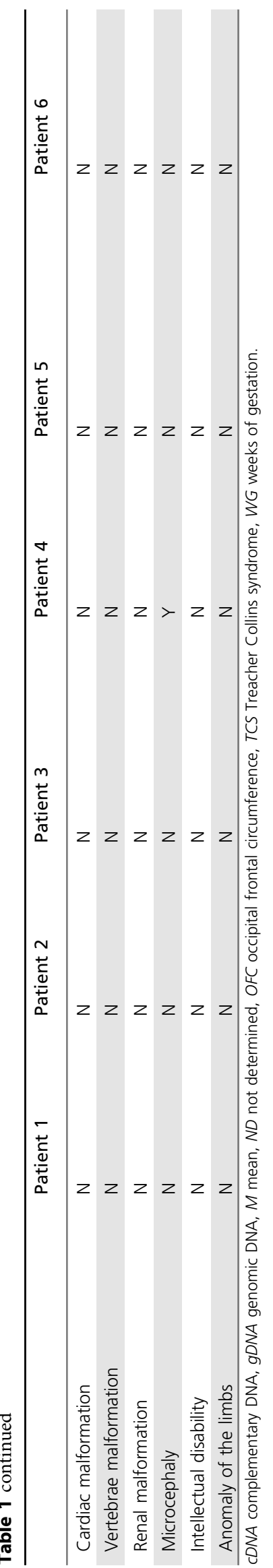

(0.1\%), and labeled for apoptosis detection by using the In Situ Cell Death Detection Kit, TMR red (Sigma), according to the manufacturer's instructions, then labeled with Hoechst. Embryos were mounted in 1\% LMT agarose for confocal microscopy observation.

\section{Molecular analyses in zebrafish}

RNA from six groups of 15 each of MO control- or MO polr1b-injected larvae at $24 \mathrm{hpf}$ were collected by using the RNeasy Mini Kit (Qiagen). The M-MLV Reverse Transcriptase kit (Invitrogen) was used to synthesize complementary DNA (cDNA) for qRT-PCR with the primers for EFla, p53, ${ }^{14} 18 \mathrm{~S}$ rRNA, 5 -ETS, ITS1, ITS2, ${ }^{15}$ and 5S. ${ }^{16}$ SYBR Green I Master reaction mix (Roche) and Rotor-Gene Q (QIAGEN's real-time PCR cycler) were used for analysis of messenger RNA (mRNA) levels of genes. Quantitative PCR (qPCR) was performed with the protocol 10 minutes at $95^{\circ} \mathrm{C}$, denaturation 10 seconds at $95^{\circ} \mathrm{C}$, annealing 10 seconds at $64^{\circ} \mathrm{C}$, and elongation 20 seconds at $72{ }^{\circ} \mathrm{C}$ (45 cycles). The relative amount of mRNA was calculated by the $2^{-\Delta \mathrm{Ct}}$ formula with EF1a as a reference.

\section{Statistical analysis}

Data were analyzed by using GraphPad Prism 6 software. Because of sample sizes $(n<30)$, the unpaired Mann-Whitney nonparametric test was used to compare two groups. GraphPad does not report variances, and standard deviations are different between the groups that are being compared. $P<0.05$ was considered statistically significant.

\section{Study approval}

Montpellier Hospital.

\section{RESULTS}

Exome sequencing identifies POLR1B as a novel candidate gene in TCS

Exome sequencing for patients 1,2 , and 3 revealed two potentially pathogenic single-nucleotide variations in POLR $1 B$ chromosome 2. In patient 1 , we identified a missense variation, c.3007C $>$ T (NM_019014.5, p.[Arg1003Cys], chr2: g.112575328C $>\mathrm{T}$, hg38), in exon 15 of POLR1B. In individuals 2 and 3 (a girl and her mother), we identified a missense variation, c.2046T $>$ A [NM_019014.5, p.(Ser682Arg), chr2: g.112568874T >A, hg38], in exon 12 of POLR1B. Both genetic variations were not found in the gnom $A D$ database. In silico analysis predicted that the genetic variants were pathogenic (Supplementary Table S1).

Via international collaboration, we found three additional individuals with TCS, and POLR1B pathogenic variants were identified by using exome and targeted sequencing. Table 1 summarizes the clinical and molecular data from these series. Pathogenic variants in POLR1B arise de novo or are inherited with a dominant pattern (Fig. 1a). One pathogenic variant (p. [Arg1003Cys], Fig. 1c) was common in three unrelated patients (patients 1,4 , and 6). 
Pathogenic variants were confirmed by PCR with exons 12 and 15 specific primers for POLR1B. Sanger results are summarized in Fig. 1c. Analysis of parental blood by PCR and Sanger sequencing determined a de novo occurrence of the pathogenic variant for patients 1,4 , and 6 . In the family of patients 2 and 3, the pathogenic variant was inherited from the affected mother. In addition, for patient 5 (c.3007C $>\mathrm{A}$, NM_019014.5, p.[Arg1003Ser], chr2:g.112575328C>A, hg38), we identified mosaicism in DNA samples isolated from the father (identified as $5^{\mathrm{m}}$ in Supplementary Fig. S1), with approximately $16 \%$ variant alleles in blood samples (15 read with pathogenic variant and 81 without pathogenic variant). Also, a mosaic POLR1B pathogenic variant was detected in DNA isolated from saliva (Supplementary Fig. S1). Of note, the father presented facial dysmorphism (slightly downslanted right eye, mild retrognathia, flat zygoma, big mouth, and thin eyelashes) but no hearing loss or coloboma.

All these data suggest that POLR1B haploinsufficiency was responsible for TCS in these five patients.

\section{POLR1B pathogenic variants affect highly conserved amino acids and probably destabilize the bridge a-helix}

To investigate the potential impact of $P O L R 1 B$ pathogenic variants on the protein conformation, we performed a conformational analysis by using computational modeling of the crystal structure of the POLR1B protein. All three pathogenic variants affected highly conserved amino acids across species (Fig. 2a). Because the crystal structure of the human protein is not available, we generated a $3 \mathrm{D}$ structure prediction based on the crystal structure of the yeast homolog protein $\mathrm{RPB}^{8}$ by using an in silico analysis described in "Materials and Methods." Both wild-type residues Ser682 and Arg1003 are located in hydrogen bonds (Fig. 2b, left panel), which participate in the stabilization of the a-helix Ser682Gln724 of POLR1B. The amino acid substitution in the model suggested that the p.(Ser682Arg) pathogenic variant might disrupt the hydrogen bond between Ser682 of the POLR1B ahelix and His967 of the POLR1A bridge helix Pro961Leu1027 (Fig. 2b, right panel), which is a highly conserved sequence located in the catalytic domain of the protein. ${ }^{8,17}$ Moreover, the p.(Arg1003Cys/Ser) pathogenic variants might destabilize the intramolecular hydrogen bond with the Gln724 residue.

\section{Polr1b expression profile and role during embryogenesis in zebrafish}

To further define the role of POLR1B during the craniofacial formation process and identify the molecular mechanisms responsible for TCS, we used a zebrafish model. First, analysis of the polr $1 b$ expression profile during zebrafish embryogenesis by in situ hybridization revealed a dynamic pattern of expression (Thisse B, Thisse C. Fast release clones: a highthroughput expression analysis. 2004. ZFIN direct data submission. https://zfin.org/ZDB-PUB-040907-1). Indeed, although polr $1 b$ was ubiquitously expressed during gastrulation from $50 \%$ epiboly to bud stages, its expression became spatially restricted during segmentation in the retina, tectum, and somite. The tissue restriction of polr $1 b$ expression continued during pharyngula to become specific to the eye, tectum, posterior myotomes, and branchial arches. Second, to examine the role of polr1b during development of the craniofacial skeleton, we used the morpholino-mediated gene knockdown strategy. The phenotype examination of zebrafish morphants generated by using a morpholino antisense oligomer that creates a steric hindrance of the ATG start codon of polr $1 b$ (MO polr $1 \mathrm{~b}$ ) revealed abnormal craniofacial development of zebrafish embryos from $20 \mathrm{hpf}$ characterized by cranioskeletal hypoplasia, microtia, and microphthalmia (Fig. 3a). Also, compared with control morphants (MO control), 72-hpf polr1b morphants were proportionally smaller and exhibited cardiac edema, altered positioning of melanocytes along the anterior-posterior axis, and pectoral fin hypoplasia (Fig. 3b).

We then focused on the development and behavior of NCCs, a migratory and multipotent cell population involved in craniofacial structures, in polr $1 b$ morphants. We injected MO polr1b in $\operatorname{Tg}($ sox 10:GFP) or $\operatorname{Tg}($ foxd3:GFP) transgenic zebrafish embryos that expressed GFP in cells positive for master neural crest lineage markers such as sox10 and foxd3, respectively. Compared with MO control heads, the smaller head observed in polr1b morphants was associated with decreased number of migrating sox $10^{+}$or foxd $3^{+}$NCCs in the head, in particular where branchial arches normally formed (Fig. 3c).

To further investigate the role of polr1b in NCC-derived craniofacial structures such as cartilaginous tissues, we injected MO polr1b in $\mathrm{Tg}$ (col2:mCherry) embryos with chondrocytes expressing mCherry and imaged them at three days postfertilization (dpf) (Fig. 3d). As revealed by image analysis and as compared with control morphants, polr $1 b$ morphants lacked mandibular and branchial arches, and the otic vesicle was smaller and misshapen (Fig. 3d). These results obtained with polr1b zebrafish morphants underline the phenotypic similarities with TCS patients showing POLR1B haploinsufficiency and suggest a new in vivo model for studying molecular mechanisms responsible for cranioskeletal defects in TCS.

\section{Mechanisms responsible for decreased number of migrating NCCs in polr1b-deficient zebrafish}

The production of ribosomes is highly conserved among species, which allows for deciphering the process in a tractable model system such as zebrafish. Ribosome biogenesis depends on RNA pol I and pol III activities that transcribe ribosomal DNA (rDNA) into $47 \mathrm{~S}$ preribosomal RNA (pre-rRNA) and $5 S$ rRNA, respectively (Supplementary Fig. S2). Thus, we used qRT-PCR to analyze the expression profile of sequences of the $47 \mathrm{~S}$ pre-rRNA including $18 \mathrm{~S}$ rRNA, the internal transcribed spacer sequences 1 and 2 (ITS1, ITS2), and the 5' external transcribed spacer (5'-ETS) in the zebrafish morphants. Compared with control morphants, polr $1 b$ morphants showed significantly decreased levels of 5'ETS, ITS1, ITS2, and 18S. In 
a
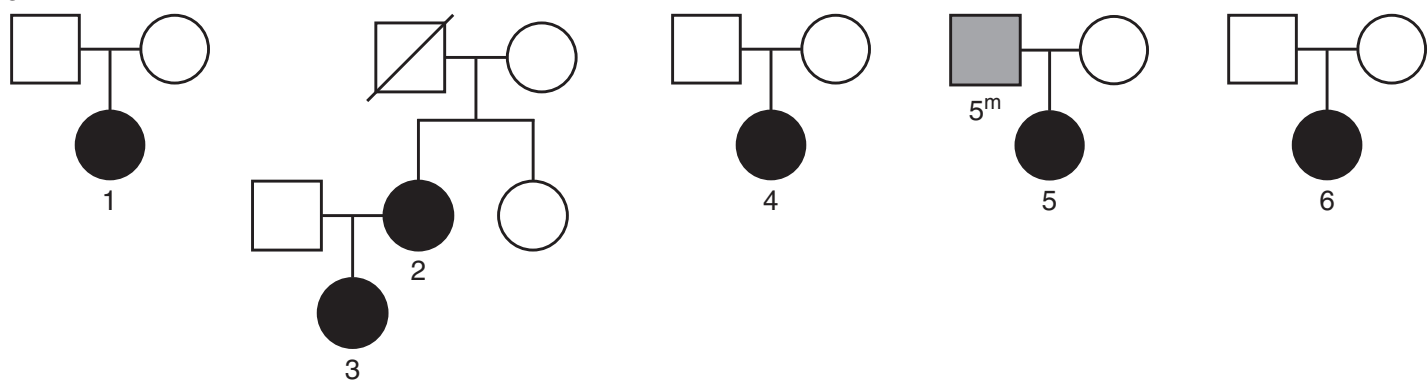

b
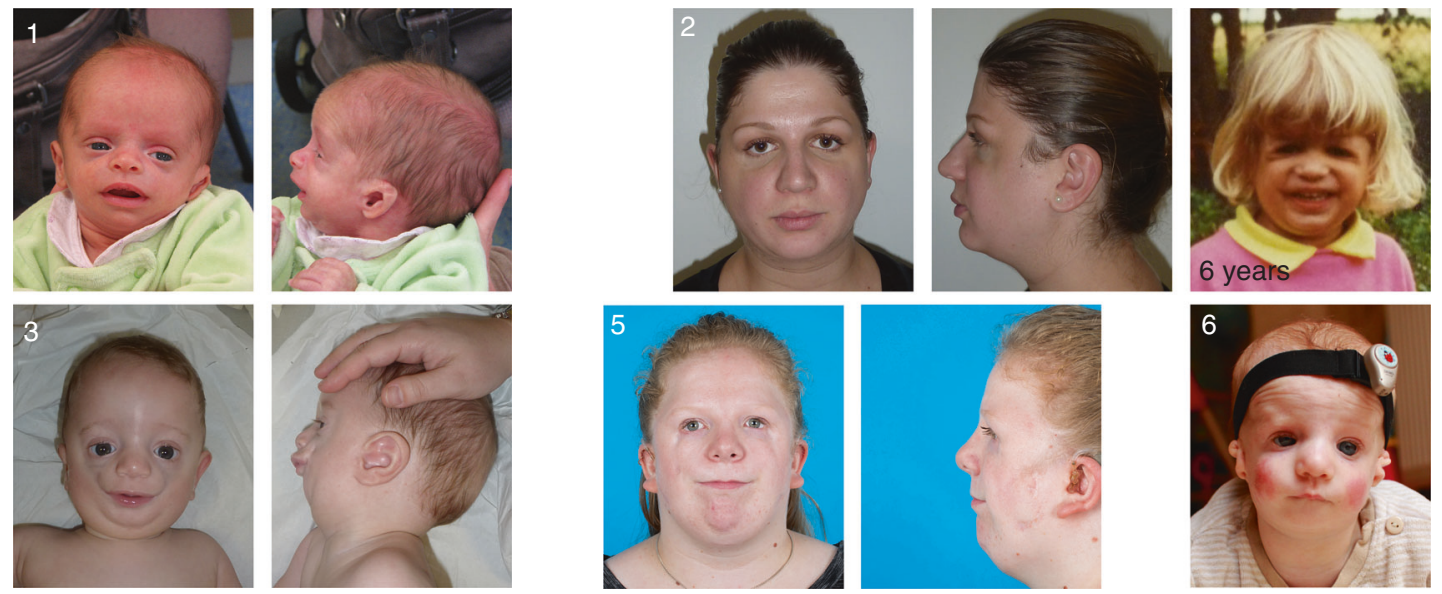

c

Patients 1, 4, 6 p.(Arg1003Cys)

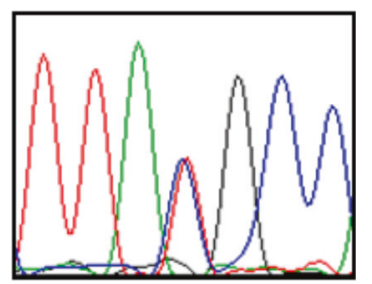

Patients 2, 3 p.(Ser682Arg)

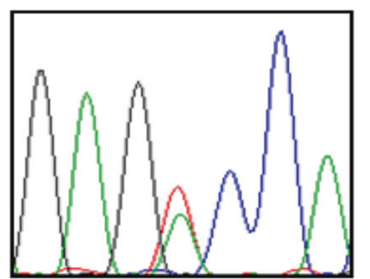

Patient 5

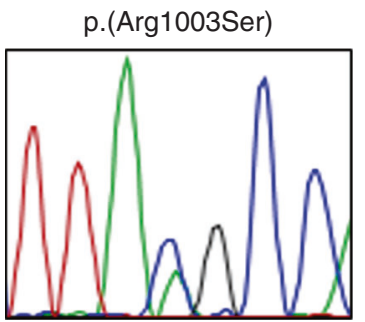

Fig. 1 POLR1B pathogenic variants in six patients with Treacher Collins syndrome (TCS). (a) Pedigrees of five families with TCS. Solid symbols indicate affected family members (patients 1-6), open symbols indicate unaffected members, gray solid symbol $\left(5^{\mathrm{m}}\right)$ indicates patient $5^{\prime} \mathrm{s}$ father with mosaicism, and squares and circles indicate males and females, respectively. (b) Front and lateral view of patients from this series. (c) Representative electropherograms of pathogenic variants.

contrast, the level of pol III-dependent 5S rRNA was similar in both morphants (Fig. 4a). Hence, polr1b knockdown did not affect pol III function, but it specifically altered pol I activity and thus the generation of $47 \mathrm{~S}$ pre-rRNA during ribosome biogenesis.

We next used the zebrafish model to further understand how polr $1 b$ deficiency and the subsequent altered pol I activity might affect the generation or migration of NCCs. Given the phenotype of polr $1 b$ morphants and that pol I is of main importance in regulating ribosome biosynthesis and protein translation, we hypothesized that POLR1B, a subunit of pol I, might play a key role in NCC viability. To detect and quantify apoptosis in zebrafish morphants, we used TUNEL assay. Confocal imaging of 20-hfp $\mathrm{Tg}($ sox $10: G F P)$ embryos revealed a higher apoptotic rate in polrlb morphants than controls (Fig. 4b-e). The massive apoptosis observed in polrlb morphants was mainly in the dorsal part of the embryos (Fig. 4d, e). Of note, sox $10^{+}$NCCs were TUNEL-negative in both polr1b morphants and controls, but massive cellular apoptosis was observed in the neuroepithelium of polr $1 b$ morphants (Fig. $4 \mathbf{b}-\mathbf{e}$ ). Therefore, the increased number of apoptotic cells in polr1b morphants did not concern NCCs that emigrate from the neural crest but rather cells of the neuroepithelium fated to differentiate into NCCs. Hence, we highlight for the first time that polr1b exhibits a nonredundant, spatiotemporal restricted function during gastrulation, which is critical for ribosome biogenesis in NCC precursors and thus their viability before NCC induction and delamination. Finally, we investigated whether the altered ribosome biogenesis caused by polrlb deficiency and the subsequent massive apoptosis observed in polr $1 b$ morphants could be associated with the level of p53 transcript. qRT-PCR revealed significantly higher p53 


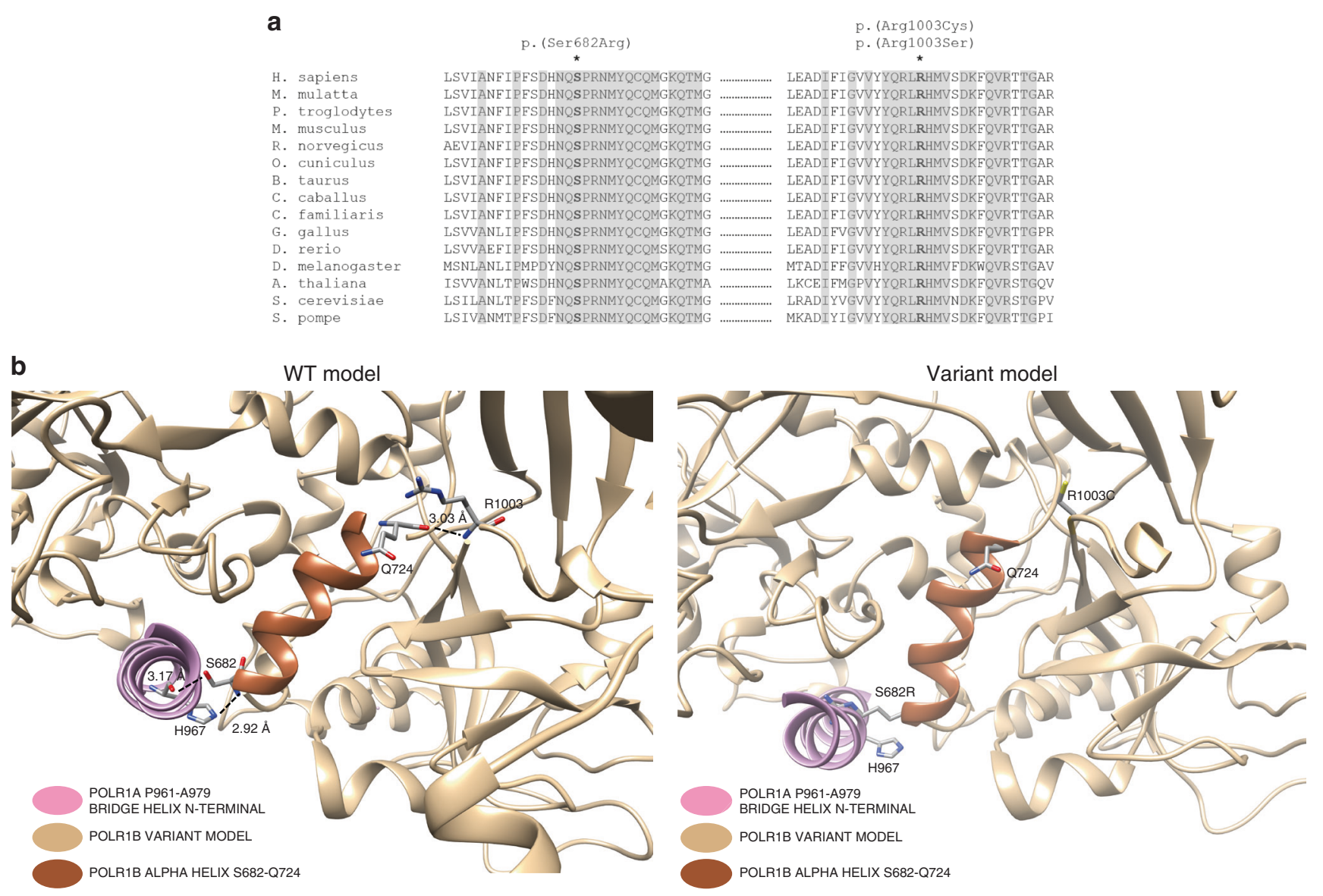

Fig. 2 In silico studies of consequences of POLR1B pathogenic variants. (a) Uniprot alignment of POLR1B protein showing interspecies conservation of amino acids affected by pathogenic variants. Position of pathogenic variants and conservation across species are highlighted in bold. (b) Space fill model of POLR1B and its association with the POLR1A subunit of RNA polymerase around the variant residues. (Left) The wild-type (WT) structure: residue found pathologically modified in Homo sapiens. Distance compatible with hydrogen bond is indicated for Ser682-His967 and Glu724-Arg1003. (Right) p. (Ser682Arg) (S682R) and p.(Arg1003Cys) (R1003C) pathogenic variant model with Arg682, which clearly could hamper the a-helix POLR1A wild-type position.

expression in 24-hpf polr1b morphants than controls at the same developmental stage (Fig. 4g).

\section{DISCUSSION}

TCS is a rare craniofacial disorder characterized by facial bone hypoplasia that results from extensive apoptosis of neuroepithelial progenitors and subsequent abnormal migration of NCCs. ${ }^{18}$ Variants in three genes (TCOF1, POLR1C, and POLR1D) have been found responsible for TCS but fail to explain all cases. In the present study, we report six TCS patients with bilateral and symmetrical malar and mandibular hypoplasia with associated facial abnormalities (microtia, coloboma, and downslanting palpebral fissures) and pathogenic variants in a novel causative gene, POLR1B. Patients with $P O L R 1 B$ pathogenic variants have craniofacial features similar to TCS patients with pathogenic variants in the other genes, although the number of reported patients is limited. We also show that, similar to TCS patients with the POLR1B pathogenic variant, polr1b knockdown in zebrafish caused severe craniofacial defects. Our results support the implication of POLR1B pathogenic variants in newly identified TCS patients.
TCS has been referred to as a heterogeneous genetic disorder caused by pathogenic variants in three different genes, which are all involved in ribosome biogenesis. Indeed, treacle, the protein encoded by TCOF1, is involved in ribosomal DNA gene transcription by interacting with the upstream binding factor, ${ }^{19}$ which is directly involved in initiating transcription of ribosomal DNA by RNA pol $\mathrm{I}^{20}$ RNA pol I is responsible for the synthesis of the $47 \mathrm{~S} / 45 \mathrm{~S}$ prerRNA, which is cleaved post-transcriptionally into $18 \mathrm{~S}, 5.8 \mathrm{~S}$, and 28S RNA molecules by small nucleolar RNAs. ${ }^{21}$ The reduction in 2'-O-methylation at nucleotide $463 \mathrm{C}$ of $18 \mathrm{~S}$ rRNA observed in Tcof1 ${ }^{+/-}$mice embryos suggests that Tcof1 might link RNA pol I-catalyzed transcription and posttranscriptional modification of pre-rRNA. ${ }^{22}$ Craniofacial structures including maxilla and mandible bones derive from the first pharyngeal arch, themselves originating from NCCs that emerge from the neuroepithelium. In mice, treacle is expressed at the edges of the neural folds and also in the developing branchial arches. ${ }^{18}$ Mouse embryos with Tcof1 haploinsufficiency show significantly reduced preribosome RNA levels as well as decreased mature ribosome number in the neuroepithelium and NCCs. ${ }^{19}$ The other genes responsible 

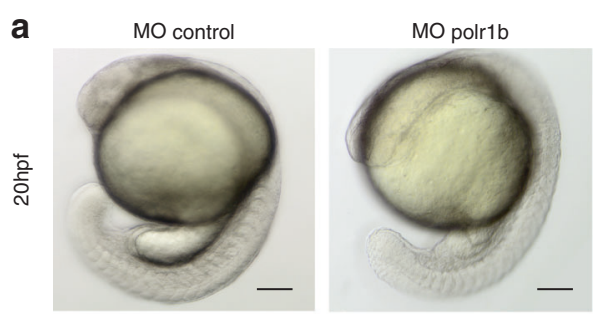

b
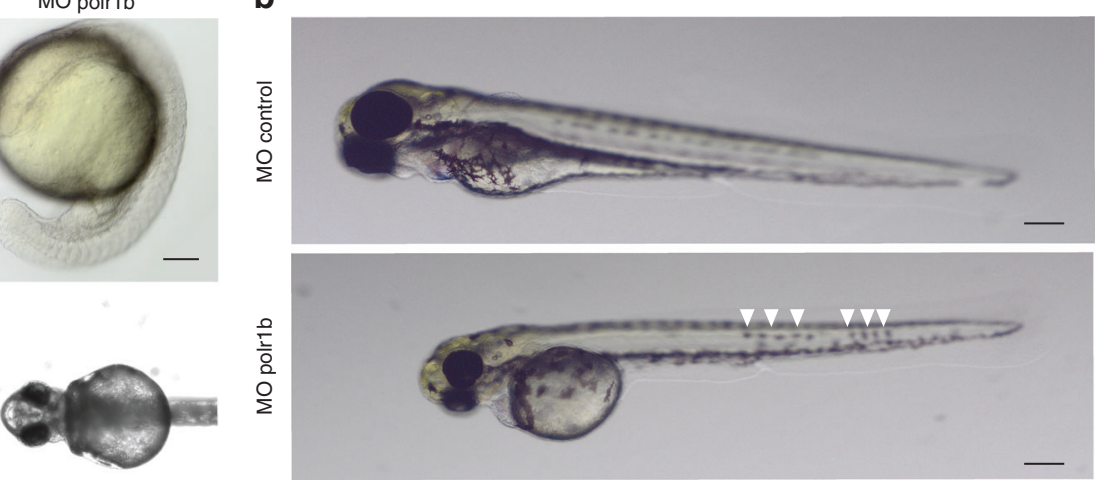

C
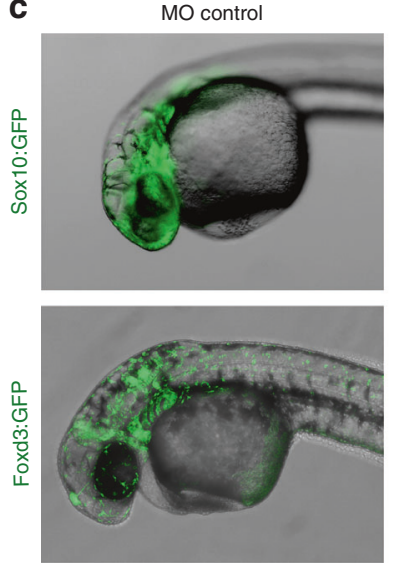
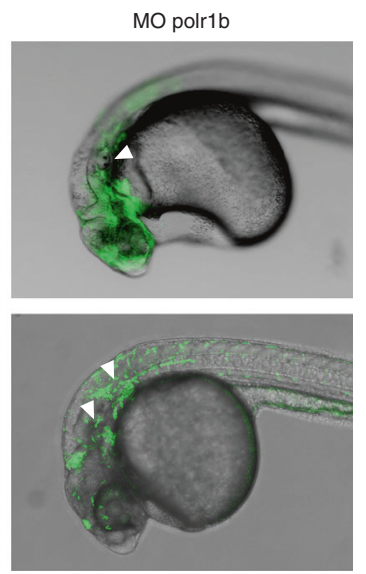

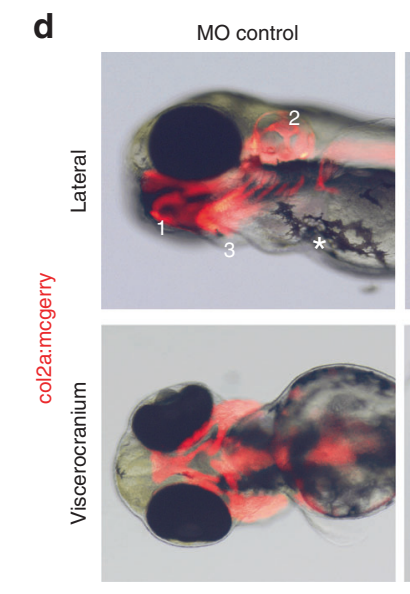

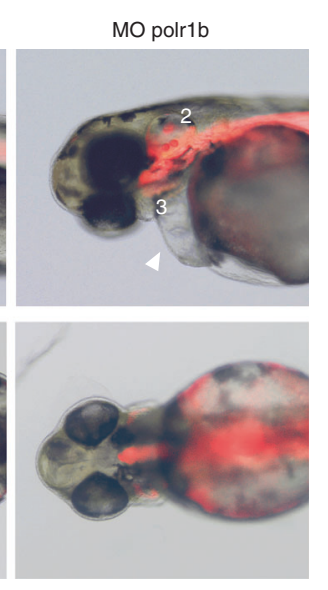

MO polr1b

Fig. 3 Polr1b knockdown in zebrafish reduces neural crest cell (NCC) migration and causes severe craniofacial defects similar to Treacher Collins phenotype. (a) At 20 hours postfertilization (hpf) (upper panels) and $48 \mathrm{hpf}$ (lower panels), polr $1 \mathrm{~b}$ morphants present smaller heads, smaller eyes, and developmental delay (larger yolk sac) compared with controls (scale bars, upper panels $=400 \mu \mathrm{m}$, lower panels $=200 \mu \mathrm{m}$ ). (b) At 3 days postfertilization (dpf), polr $1 b$ morphants present smaller size as well as pigmentation defects (indicated by white arrows) compared with controls, and some morphants also present cardiovascular defects (large cardiac edema) and died at $4 \mathrm{dpf}$ (data not shown). (c) polr1b morphants present altered NCC marker expression. At $30 \mathrm{hpf}$, injection of $\operatorname{Tg}($ sox 10:GFP) (upper panels) and $T g(f o x d 3: G F P)$ (lower panels) conferred NCC abnormalities in polr1b morphants compared with controls, in particular where branchial arches normally formed (white arrows, right panels), which indicates reduced migratory NCC population. (d) At 3 dpf, cranioskeletal structures were visualized by using Tg(col2a:mCherry). Compared with control morphants (MO Control), polr1b morphants (MO polr $1 \mathrm{~b}$ ) lacked mandibular and branchial arches (1: mandibula, 3: branchial arches), and the otic vesicle was smaller (2). polr1b morphants show impaired pigmentation (pigments indicated by an asterisk) and cardiac edema (white arrow, upper right panel), which indicates that other NCC-derived tissues are affected. Scale bars $=200 \mu \mathrm{m}$. Five embryos were used in each condition and representative images of six experiments are shown.

for TCS, namely POLR1C and POLR1D, ${ }^{5}$ encode subunits that belong to the 14-subunit RNA pol I protein. ${ }^{23}$ Like TCOF1, the genes POLR1C and POLR1D disturb the biogenesis of ribosomes as well as NCC generation and survival, causing TCS when presenting pathogenic variation. ${ }^{3}$ Additionally, POLR1A encoding for the largest subunit of RNA pol I was found responsible for an acrofacial dysostosis named acrofacial dysostosis Cincinnati type. ${ }^{14}$

Our study expands on the genotypic and phenotypic heterogeneity of congenital acrofacial disorders resulting from ribosome biogenesis disruption. In line with previous reports, we show that polrlb knockdown in zebrafish was associated with a deficit of rRNA transcripts specifically dependent on RNA pol I. Also, this polrlb knockdown induced massive apoptosis in the neuroepithelium at the NCC formation stage, which was accompanied by increased p53 expression. Similarly, the zebrafish polrla variant shows deficiencies in the biogenesis of ribosomes leading to extended death in the neuroepithelium and subsequent cranioskeletal malformation mimicking TCS. ${ }^{14}$ In addition, zebrafish studies demonstrated that pathogenic variants in polr1c and polr1d lead to craniofacial features mimicking defects observed in TCS. ${ }^{3}$ Impaired ribosome biogenesis can cause activation of p53, leading to cell cycle arrest. ${ }^{24}$ In a study using the Tcof1 variant mouse, inhibition of $\mathrm{p} 53$ prevented TCS. ${ }^{25}$ From these studies and our data, we propose that in TCS patients with pathogenic variants in POLR1B, massive p53-dependent apoptosis of cells within the neuroepithelium is responsible for the poor number of migrating NCCs and the cranioskeletal malformations.

Considering that haploinsufficiency of Tcofl, or other genes belonging to the RNA pol I macromolecule, is sufficient to lead to neuroepithelium and NCC death during development, TCS 

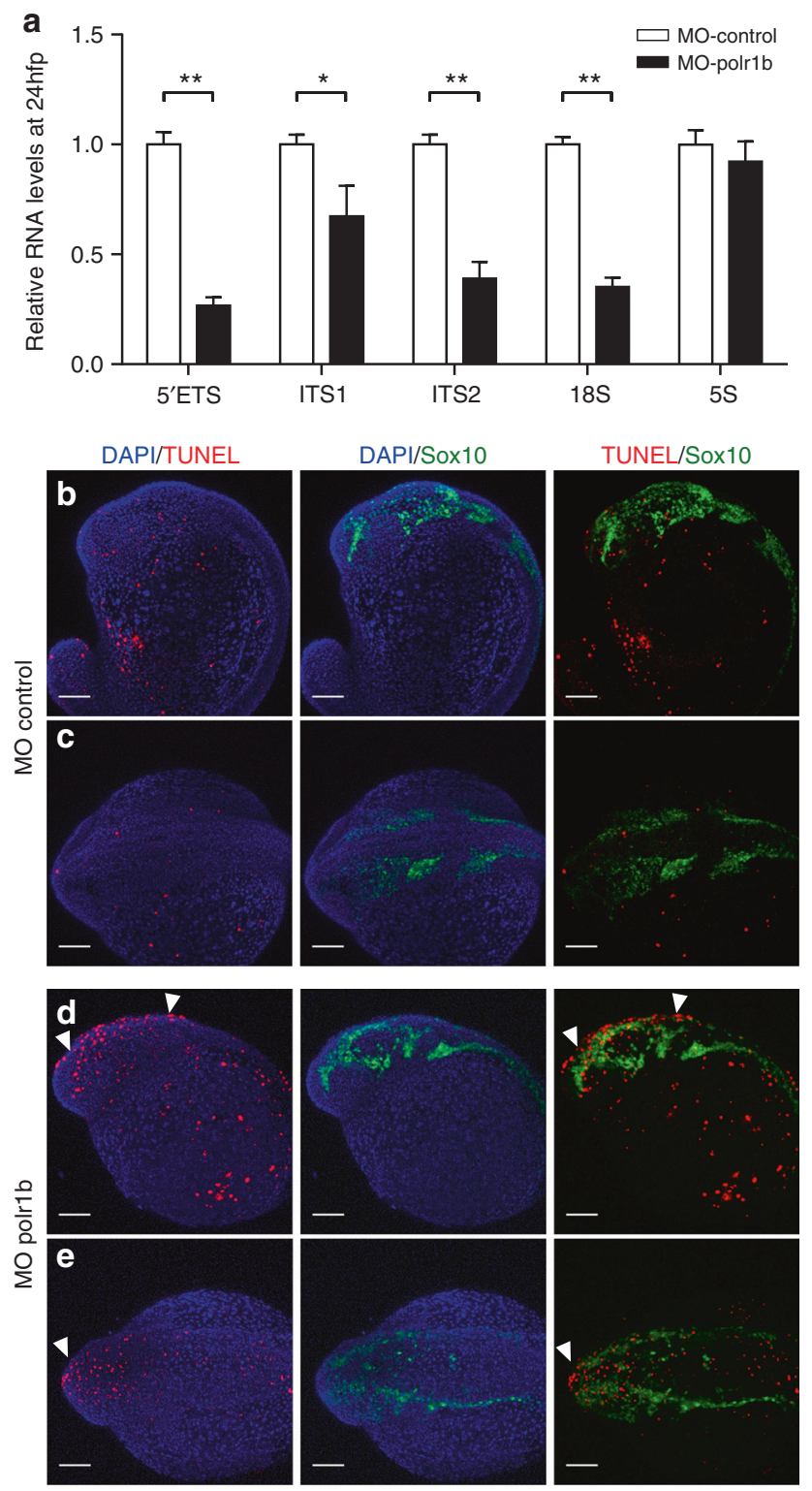

f
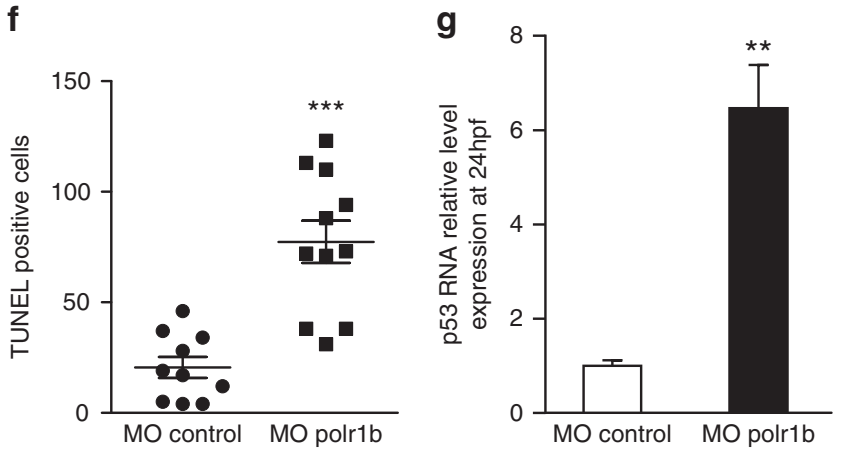

could be explained by increased sensitivity of the neuroepithelium and NCCs to cell stress (here ribosome biogenesis deficiency) at this specific time of development. During embryogenesis in zebrafish, the expression of polrlb is ubiquitous at early stages and becomes progressively restricted. At the pharyngula stage, polr $1 b$ expression is restricted to
Fig. 4 Polr1b knockdown in zebrafish alters RNA polymerase I (pol I)-mediated ribosome biogenesis in neural crest cell (NCC) precursors. (a) Ribosomal RNA (rRNA) transcript expression profile in control and polr $1 \mathrm{~b}$ morphants by quantitative reverse transcription polymerase chain reaction (qRT-PCR) normalized to EF1a level. 5'ETS, ITS1, ITS2, and $18 \mathrm{~S}$ depend on RNA pol I activity, whereas $5 \mathrm{~S}$ is RNA pol III-dependent. (be) Confocal images of terminal deoxynucleotidyl transferase dUTP nick end labeling (TUNEL) labeling (in red) of $20 \mathrm{hpf}$ Tg(sox10:GFP) embryos (lateral views in (b,d), dorsal views in $(\mathbf{c}, \mathbf{e})$ ). Imaging of apoptotic cells stained with TUNEL in polr $1 \mathrm{~b}$ and control morphants (dorsal part indicated by white arrows in (d,e)). Scale bar: $100 \mu \mathrm{m}$. (f) Quantification of TUNEL-positive cells in $\mathrm{MO}$ control and $\mathrm{MO}$ polr1b $(n=10$ zebrafish larvae in MO control and $n=11$ zebrafish larvae in MO polr1b). (g) Quantification of p53 messenger RNA (mRNA) level in MO control and MO polr $1 \mathrm{~b}$ ( $n=6$ groups of 15 pooled zebrafish larvae at $24 \mathrm{hpf})$. Data are mean \pm SEM. ${ }^{*} p<0.05,{ }^{* *} p<0.01$, ${ }^{* * *} p<0.001$ by one-tailed unpaired Mann-Whitney nonparametric test.

pharyngeal arches, which suggests a role for polrlb in these NCC derivatives. However, in line with Tcofl studies in mice $^{19,25}$ and studies of RNA pol I subunits ${ }^{3,14}$ in zebrafish, we showed that NCC apoptosis occurs in the neural tube and not in migrating NCCs or in NCC derivatives in which we observed this restricted expression of polr1b. Although the expression patterns of the different RNA pol I subunits in tissues originating from the neural crest remain unexplored during human embryo development, one interesting research line for future studies would be to determine a possible association with extended neuroepithelium cell death in TCS.

Finally, our conformational analysis based on the crystal structure of the homologous yeast protein showed that all three pathogenic variants affected amino acid residues involved in hydrogen bond interactions with the a-helix Ser682-Gln724 of POLR1B. Importantly, the pathogenic variant affecting Ser682 might introduce a steric constraint on the helix position and directly affect the interaction between the POLR1B a-helix and the N-terminal portion of the bridge helix of POLR1A. The bridge helix domain is a central component of the catalytic site of all cellular RNA polymerases (RNAPs). It coordinates the movement of several other domains during catalysis and is involved in all known functions of RNAPs. ${ }^{17}$ High-throughput mutagenesis studies of the Methanocaldococcus jannaschii bridge helix domain showed that pathogenic variants affecting this domain, and in particular the molecular hinge region in the $\mathrm{N}$-terminal portion of the bridge helix, severely reduced RNAP enzymatic activity. ${ }^{26}$ In particular, every pathogenic variant on His808, homolog of His967 in POLR1A, induces a strong decrease in catalytic activity. We hypothesized that the p.(Ser682Arg) pathogenic variant could produce the same loss of function. Both pathogenic variants affecting the amino acid residue at position 1003 might disrupt the hydrogen intramolecular interaction with the a-helix Ser682-Gln724 of POLR1B and indirectly destabilize the bridge helix of POLR1A, thus similarly reducing RNAP activity of the $H$. sapiens protein.

In conclusion, TCS so far belongs to the largest group of diseases named ribosomopathies (e.g., Diamond-Blackfan 
syndrome), ${ }^{27}$ and our present work adds $P O L R 1 B$ to the list of genes (TCOF1, POLR1A, POLR1C, and POLR1D) already known to be crucial for ribosome biogenesis and NCC survival. The proportion of TCS attributed to pathogenic variants in TCOF1, POLR1C, and POLR1D is $63-93 \%, 6 \%$, and $1.2 \%$, respectively. ${ }^{28}$ Because the POLR $1 B$ pathogenic variants we describe diagnoses $1.3 \%$ of the $4 \%$ of individuals with typical clinical signs of TCS but without a molecular diagnosis, our study has important consequences for genetic diagnosis of TCS.

\section{SUPPLEMENTARY INFORMATION}

The online version of this article (https://doi.org/10.1038/s41436019-0669-9) contains supplementary material, which is available to authorized users.

\section{ACKNOWLEDGEMENTS}

We thank the patients and the family members for their support. Part of this work was supported by the French Franceschetti-Treacher Collins association Coline (http:// netcoline.org/), the research program Programme Hospitalier de Recherche Clinique Régional Languedoc-Roussillon and the Plan National Maladies Rares 2011-2014 from the French Direction Générale de l'Organisation des Soins (DGOS). This work was supported by the Institut National de la Santé et Recherche Médicale (INSERM) and the University of Montpellier.

\section{DISCLOSURE}

The authors declare no conflicts of interest.

Publisher's note Springer Nature remains neutral with regard to jurisdictional claims in published maps and institutional affiliations.

\section{REFERENCES}

1. Fazen LE, Elmore J, Nadler HL. Mandibulo-facial dysostosis. (TreacherCollins syndrome). Am J Dis Child. 1967;113:405-410.

2. Werner A, Iwasaki S, McGourty CA, et al. Cell-fate determination by ubiquitindependent regulation of translation. Nature. 2015;525:523-527.

3. Noack Watt KE, Achilleos $A$, Neben $C L$, et al. The roles of RNA polymerase I and III subunits Polr1c and Polr1d in craniofacial development and in zebrafish models of Treacher Collins syndrome. PLoS Genet. 2016;12:e1006187.

4. Positional cloning of a gene involved in the pathogenesis of Treacher Collins syndrome. The Treacher Collins Syndrome Collaborative Group. Nat Genet. 1996;12:130-136.

5. Dauwerse JG, Dixon J, Seland $S$, et al. Mutations in genes encoding subunits of RNA polymerases I and III cause Treacher Collins syndrome. Nat Genet. 2011;43:20-22.

6. Vincent M, Genevieve D, Ostertag A, et al. Treacher Collins syndrome: a clinical and molecular study based on a large series of patients. Genet Med. 2016;18:49-56.

7. Sobreira N, Schiettecatte F, Boehm C, et al. New tools for Mendelian disease gene identification: PhenoDB variant analysis module; and GeneMatcher, a web-based tool for linking investigators with an interest in the same gene. Hum Mutat. 2015;36:425-431.

8. Fernandez-Tornero C, Moreno-Morcillo M, Rashid UJ, et al. Crystal structure of the 14-subunit RNA polymerase I. Nature. 2013;502:644-649.

9. Nguyen-Chi M, Laplace-Builhe B, Travnickova J, et al. Identification of polarized macrophage subsets in zebrafish. eLife. 2015;4:e07288.
10. Gilmour DT, Maischein HM, Nusslein-Volhard C. Migration and function of a glial subtype in the vertebrate peripheral nervous system. Neuron. 2002;34:577-588.

11. Carney TJ, Dutton KA, Greenhill E, et al. A direct role for Sox10 in specification of neural crest-derived sensory neurons. Development. 2006;133:4619-4630.

12. Mitchell RE, Huitema LF, Skinner RE, et al. New tools for studying osteoarthritis genetics in zebrafish. Osteoarthritis Cartilage. 2013;21:269-278.

13. Nguyen-Chi M, Phan QT, Gonzalez C, et al. Transient infection of the zebrafish notochord with E. coli induces chronic inflammation. Dis Model Mech. 2014;7:871-882.

14. Weaver KN, Watt KE, Hufnagel RB, et al. Acrofacial dysostosis, Cincinnati type, a mandibulofacial dysostosis syndrome with limb anomalies, is caused by POLR1A dysfunction. Am J Hum Genet. 2015;96:765-774.

15. Azuma M, Toyama R, Laver E, Dawid IB. Perturbation of rRNA synthesis in the bap28 mutation leads to apoptosis mediated by p53 in the zebrafish central nervous system. J Biol Chem. 2006;281:13309-13316.

16. Diaz de Cerio O, Rojo-Bartolome I, Bizarro C, et al. 5S rRNA and accompanying proteins in gonads: powerful markers to identify sex and reproductive endocrine disruption in fish. Environ Sci Technol. 2012;46:77637771.

17. Weinzierl RO. The Bridge Helix of RNA polymerase acts as a central nanomechanical switchboard for coordinating catalysis and substrate movement. Archaea. 2011;2011:608385.

18. Dixon J, Jones NC, Sandell LL, et al. Tcof1/Treacle is required for neural crest cell formation and proliferation deficiencies that cause craniofacial abnormalities. Proc Natl Acad Sci U S A. 2006;103:13403-13408.

19. Valdez BC, Henning D, So RB, et al. The Treacher Collins syndrome (TCOF1) gene product is involved in ribosomal DNA gene transcription by interacting with upstream binding factor. Proc Natl Acad Sci U S A. 2004;101:10709-10714.

20. Goodfellow SJ, Zomerdijk JC. Basic mechanisms in RNA polymerase I transcription of the ribosomal RNA genes. Subcell Biochem. 2013;61:211-236.

21. Moore PB, Steitz TA. The involvement of RNA in ribosome function. Nature. 2002;418:229-235.

22. Gonzales B, Henning D, So RB, et al. The Treacher Collins syndrome (TCOF1) gene product is involved in pre-rRNA methylation. Hum Mol Genet. 2005;14:2035-2043.

23. Kuhn $C D$, Geiger SR, Baumli $S$, et al. Functional architecture of RNA polymerase I. Cell. 2007;131:1260-1272.

24. Pestov DG, Strezoska Z, Lau LF. Evidence of p53-dependent cross-talk between ribosome biogenesis and the cell cycle: effects of nucleolar protein Bop1 on G(1)/S transition. Mol Cell Biol. 2001;21:4246-4255.

25. Jones NC, Lynn ML, Gaudenz K, et al. Prevention of the neurocristopathy Treacher Collins syndrome through inhibition of p53 function. Nat Med. 2008;14:125-133.

26. Weinzierl RO. The nucleotide addition cycle of RNA polymerase is controlled by two molecular hinges in the Bridge Helix domain. BMC Biol. 2010;8:134.

27. Mills EW, Green R. Ribosomopathies: there's strength in numbers. Science. 2017;358:eaan2755.

28. Katsanis SH, Jabs EW. Treacher Collins syndrome. In: Adam MP, Ardinger HH, Pagon RA, Wallace SE, Bean LH, Stephens K, Amemiya A, (eds) GeneReviews. Seattle, WA: University of Washington-Seattle; 2004.

\section{(i) $(2)$}

Open Access This article is licensed under a Creative Commons Attribution-NonCommercial-NoDerivatives 4.0 International License, which permits any non-commercial use, sharing, distribution and reproduction in any medium or format, as long as you give appropriate credit to the original author(s) and the source, and provide a link to the Creative Commons license. You do not have permission under this license to share adapted material derived from this article or parts of it. The images or other third party material in this article are included in the article's Creative Commons license, unless indicated otherwise in a credit line to the material. If material is not included in the article's Creative Commons license and your intended use is not permitted by statutory regulation or exceeds the permitted use, you will need to obtain permission directly from the copyright holder. To view a copy of this license, visit http://creativecommons.org/licenses/by-nc-nd/4.0/.

(c) The Author(s) 2019 